\title{
Jetzt gemeinsam: JHaS und Swiss Young Internists
}

\author{
Agieren statt reagieren, so lautet das Motto des Vereins «Junge Hausärztinnen und \\ -ärzte Schweiz (JHaS)». Die Gründung einer neuen Fachgesellschaft und von «Swiss \\ Young Internists» war Anlass zu einem Treffen. Beide Gruppierungen streben die \\ Zusammenarbeit an, um gemeinsame Ziele zu verfolgen, zum Beispiel die Webseite \\ myAIM.
}

\section{Sven Streit ${ }^{a}$ \\ Ewelina Biskup ${ }^{b}$ \\ a Präsident JHaS \\ b Vorsitzende Swiss Young Internists}

Ärzten wird gelegentlich vorgeworfen, auf sich allein gestellt etwas «eigenbrötlerisch» zu handeln. Es ist aber auch zu beobachten, dass Ärzte vernetzt arbeiten, Gruppen bilden, Verbände gründen und so ihre gemeinsamen Interessen vertreten. Die Zusammenarbeit gründet dabei zunächst auf gemeinsamen Werten, dann auf gemeinsamen Zielen und später auch auf gemeinsamen Strukturen. Auch die Gründung des Vereins «Junge Hausärztinnen und -ärzte Schweiz (JHaS)» entstand auf diese Weise vor acht Jahren, angeregt durch die europäische Jungärzteorganisation (VdGM). Gemeinsame Ziele entwickeln wir seither jährlich an einer Retraite - so auch am 23. und 24. November 2013. Und so entstanden nach und nach eine Struktur, der Verein, ein eigener Kongress. Das jährliche Überdenken ist wichtig, um nicht plötzlich ohne Inhalt, mit divergierenden Zielen oder mit einer nicht mehr zeitgemässen Struktur dazustehen. Wer möchte schon in einer Organisa-

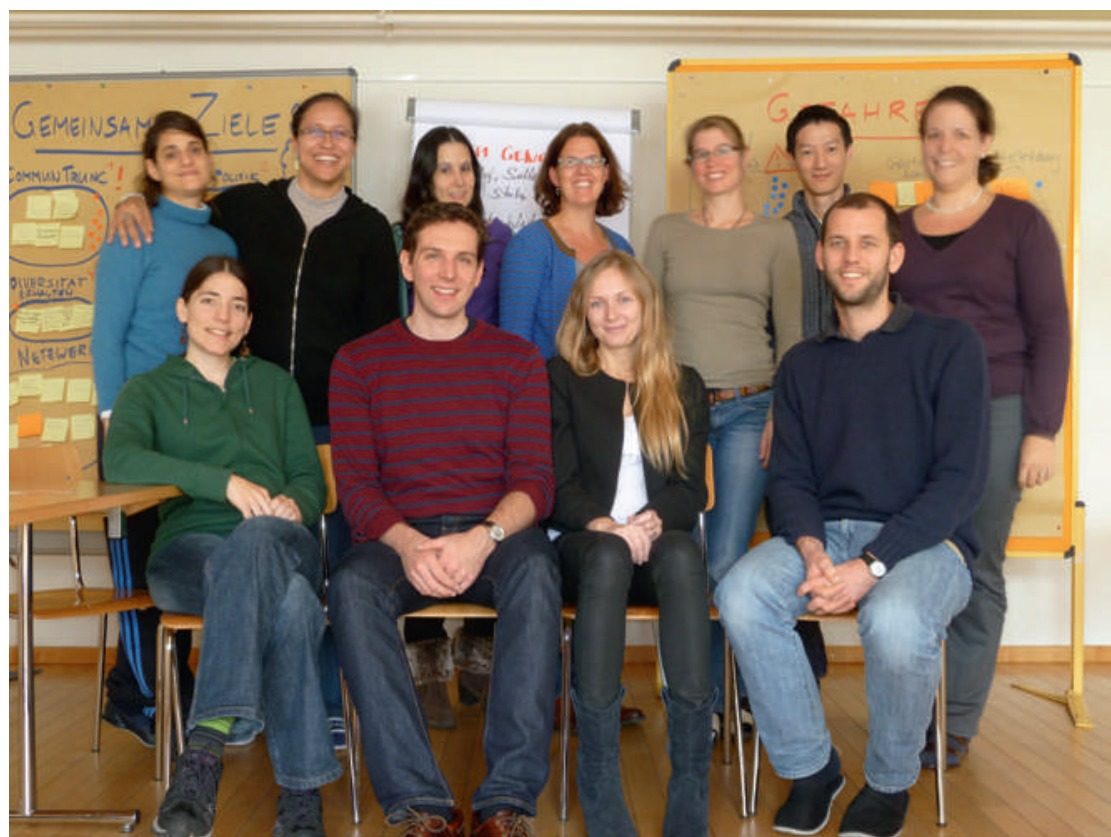

Die Vertreter von «Junge Hausärztinnen und -ärzte Schweiz» sowie von «Swiss Young Internists» bei ihrem Treffen zur Vereinbarung der Zusammenarbeit. tion Mitglied sein, die es a) nicht mehr braucht, b) keine Ziele mehr hat und c) nur noch aus historischen Gründen existiert? Nun haben wir es in acht Jahren erwartungsgemäss nicht geschafft, die fehlende Generation an Hausärzten zwischen den jetzt in Pension Tretenden und den Berufseinsteigern aus dem Hut zu zaubern. Trotzdem erleben wir ein Revival der Hausarztmedizin, wie die zahlreichen Aussagen von Arbeitskollegen zeigen, die später in einer Praxis arbeiten möchten. Am JHaS-Kongress erleben wir die neue Aufbruchstimmung, die uns in der Vereinsarbeit sehr motiviert. Was wollen wir also noch erreichen?

In zwei Jahren entsteht aus den zwei grössten Fachgesellschaften SGAM (Schweizerische Gesellschaft für Allgemeinmedizin) und SGIM (Schweizerischen Gesellschaft für Allgemeine Innere Medizin) eine neue Gesellschaft für Allgemeine Innere Medizin, wobei der endgültige Namen noch unklar ist. Diesen Prozess möchten wir unbedingt unterstützen und proaktiv sein. Als junge Generation angehender Fachärztinnen und -ärzte für Allgemeine Innere Medizin sehen wir uns in der Verantwortung, die bewährte Zusammenarbeit zwischen ambulanten und spitalinternen Generalisten zu stärken. Unser oberstes Ziel ist die patientenzentrierte, sinnvolle und qualitativ hochstehende Versorgung der Patientinnen und Patienten. Dafür brauchen wir eine starke Fachgesellschaft, die unsere gemeinsame Kultur des Generalismus pflegt und den Nachwuchs fördert.

Das gemeinsame Weiterbildungsprogramm haben wir zunächst als einseitig und zu eng gefasst empfunden. Unsere Kritik wurde wahrgenommen, und so sehen wir das Weiterbildungsprogramm heute als gemeinsame Akademie für angehende Generalisten in Praxis und Spital. Es gibt gemeinsame Facharztprüfungen und bald gemeinsame Fortbildungsprogramme. Wir erfahren einen offenen und motivierenden Dialog mit den beiden Gesellschaften und ihren Präsidenten, die die Zeichen der Zeit erkannt haben. Hinderliche Diskrepanzen werden erfolgreich abgebaut. 


\section{Was bedeutet die neue Fachgesellschaft für die JHaS?}

Diese Frage und daraus abgeleitet die Zukunftsideen für die JHaS besprachen wir mit Ewelina Biskup, der designierten Vertreterin der Swiss Young Internists auf dem verschneiten Moeschberg ob Bern. Zur Vorbereitung trafen sich beide Vertreter separat, um die gemeinsamen Schritte im Vorfeld zu besprechen. Die JHaS traf auf eine neue, offene und zur Zusammenarbeit motivierte Organisation, die viele Parallelen in ihrer Entstehung aufweisen: Beide Gruppen entwickelten sich aus den entsprechenden Jungorganisationen Europas (VdGM führte zu JHaS, youngEFIM zu Swiss Young Internists), beide wurden durch die nationalen Gesellschaften unterstützt, beide sind sowohl landes- als auch europaweit aktiv und vertreten die Schweiz in einem globalen Netzwerk. Und nicht zuletzt: Beide Organisationen repräsentieren die in der Schweiz durch die Zusammenarbeit von Spitalärzten und Praxisärzten existierende ganz spezielle Situation.

\section{Gemeinsam Ziele erreichen}

In einem ersten Schritt wollten wir unsere jeweilige Organisation besser kennenlernen, bevor wir dann gemeinsame Ziele festlegten. Rasch entstand eine Welt gemeinsamer Werte: Über allem - und doch von allen manchmal vernachlässigt in den Diskussionen - steht der Anspruch einer guten medizinischen Versorgung unserer Patienten zu Hause und im Spital. Darauf stützt sich unser Wunsch, vor lauter Spezialisierung nicht den Generalismus zu vernachlässigen, sondern zu fördern. Dafür wollen wir uns für einen starken «Common track» in der Weiterbildung engagieren. Weiter möchten wir als Netzwerk junge Leute an die richtige Stelle bringen und uns für eine starke Politik in der Versorgung unserer Patienten einsetzen. Natürlich sind uns dabei die Nachwuchsförderung und die gemeinsame Forschung zur Akademisierung sowie eine europaweite und globale Vernetzung genauso wichtig. Neben der

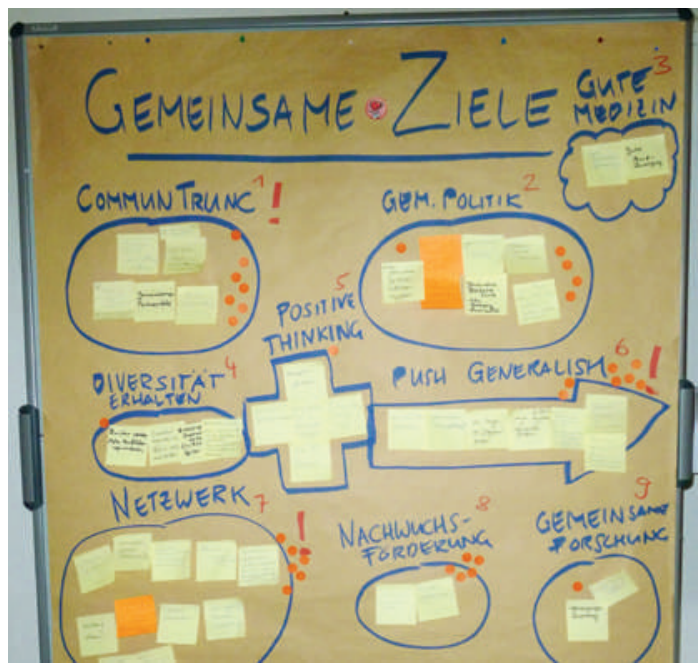

guten Versorgung möchten wir aber vor allem durch positives Handeln auffallen und uns neben gemeinsamen Zielen auch für die Diversität unserer Berufe einsetzen.

\section{Kollaboration und Erhalt verschiedener Profile}

So weit also herrscht Einigkeit. Wir, JHaS und Swiss Young Internists, wollen dem Beispiel unserer «Seniors» folgen, und eng zusammenarbeiten.

Gab es keine Dissonanzen? Zurzeit und speziell auf persönlicher Ebene nicht, aber als Brainstorming haben wir uns überlegt, welche individuellen Interessen der Einzelnen zu beachten sind. Ein häufig genanntes Votum betraf die eigene Identität: das Image, das wir für die künftige Generation der Hausärzte sehen. Ist es dasselbe wie für Spitalärzte? Sicherlich bedarf es einer soliden Arbeit, das Profil beider Berufsfelder zu wahren und die Charakteristika zu konsolidieren. Des Weiteren sollen selbstverständlich spezifische Interessen (z. B. Praxisassistenz, Stopp von unnötigen und teuren Zusatzqualifikationen neben dem Facharzttitel) weiterverfolgt werden.

Basierend auf diesen gemeinsamen Werten entschieden sich die JHaS und Swiss Young Internists, gemeinsam zusammenzuarbeiten. Ein erstes Projekt ist die Webseite myAIM (s. Kasten).

Wir freuen uns über Ihre Unterstützung!

\section{Vorankündigung}

myAIM - Die neue Webseite für angehende Fachärztinnen und -ärzte für Allgemeine Innere Medizin

Wir haben ein gemeinsames Ziel: die breite und gute ärztliche Versorgung unserer Patienten in Praxis und Spital. Dafür gibt es seit 2011 einen gemeinsamen Facharzttitel AIM. Wir, die unten stehenden Gesellschaften, werden nächstes Jahr eine Webseite aufbauen, um Informationen zusammenzutragen, die angehende Fachärzte brauchen. Welche Kurse muss ich besuchen? Wie bereite ich mich für die Prüfung vor? Wo finde ich eine attraktive Stelle? Welche Forschung in Allgemeiner Innerer Medizin gibt es? Welche Kongresse könnten mich interessieren? Was muss ich bei der Weiterbildung beachten? Das Gratisangebot richtet sich an Assistenzärztinnen und -ärzte. Wer sich auf myAIM registriert, erhält nach seinen Wünschen die gesuchten Informationen wie z.B. Kurse in seiner Region.

myAIM - mein Ziel: Allgemeine Innere Medizin Ab 2014 auf www.myaim.ch - Eine Kooperation von: JHaS \& Swiss Young Internists sowie SGAM \& SGIM. Unterstützt durch: BAG, CRMF, SIWF, WHM und die fünf Institute für Hausarztmedizin. 